\title{
Common mechanism of pathogenesis in various types of metastatic osteosarcoma
}

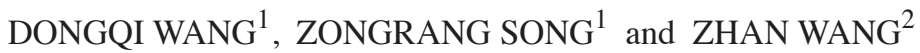 \\ Departments of ${ }^{1}$ Spinal Surgery and ${ }^{2}$ Traumatic Orthopedics, Xi'an Jiaotong University, \\ Medical College Red Cross Hospital, Xi'an, Shaanxi 710054, P.R. China
}

Received November 18, 2015; Accepted April 26, 2017

DOI: $10.3892 / 01.2017 .6955$

\begin{abstract}
The present study aimed to investigate the common metastatic mechanism in various types of metastatic osteosarcoma (OS). Gene expression profiles generated from the metastatic OS KHOS and KRIB cell lines and the non-metastatic OS HOS cell line were compared. Two groups of differentially expressed genes (DEGs) between KHOS or KRIB and HOS were screened $(\mathrm{P}<0.01$ and Ifold changel $\geq 2$ ) and then underwent Gene Ontology (GO) and pathway enrichment analyses. Subsequently, the protein-protein interaction (PPI) network was constructed and the subnetwork was mined. Furthermore, overlapping DEGs of these two groups were identified and pathway enrichment and regulatory network analyses were performed. A total of 1,552 and 1,330 DEGs from KHOS vs. HOS and KRIB vs. HOS were obtained, respectively. GO and pathway enrichment analyses of DEGs between KRIB and HOS, including anatomical structure morphogenesis and focal adhesion, were similar to those between KHOS and HOS. Vascular endothelial growth factor A and epidermal growth factor receptor were hub nodes in the PPI network for KHOS and KRIB. Subnetworks of these two groups were similar. In addition, 421 upregulated and 595 downregulated overlapping genes were enriched in the mitogen-activated protein kinase and transforming growth factor- $\beta$ signaling pathways. Furthermore, seven vital transcription factors, including hes-related family bHLH transcription factor with YRPW motif 1 (HEY1), were obtained. Overall, different types of metastatic OS were shown to exhibit a similar mechanism of pathogenesis. With the exception of cell adhesion and angiogenesis, recapitulation of the morphogenetic processes facilitates OS tumor formation and metastasis. Genes such as HEY1 are important for metastatic
\end{abstract}

Correspondence to: Dr Zhan Wang, Department of Traumatic Orthopedics, Xi'an Jiaotong University, Medical College Red Cross Hospital, 555 Friendship Road, Xi'an, Shaanxi 710054, P.R. China E-mail: zhan_wangzw@163.com

Key words: metastatic osteosarcoma, differentially expressed genes, protein-protein interaction network, module
OS. Further studies are required in order to confirm these results.

\section{Introduction}

Osteosarcoma (OS), which is one of the common primary bone tumors in children and adolescents is highly aggressive and metastatic (1); it comprises $\sim 5 \%$ of all pediatric tumors and patients with pulmonary metastasis present a poor prognosis (2). There are definite differences in the mechanism between primary and metastatic OS, and numerous previous studies have been performed to expound them (3-5).

Multiple signaling pathways, including the Notch signaling pathway, are involved in cancer migration initiation through the extracellular matrix (ECM) towards the bloodstream (6). Survival of cancer cells in the bloodstream is mediated by the integrin signaling pathway to activate the phosphatidylinositol 3-kinase/protein kinase B survival pathway (7). Signaling pathways, including those of Src and Wnt, are involved in apoptosis resistance throughout metastasis $(8,9)$. In addition, the immune system is also invaded and modulated for survival advantage (10). Subsequently, cell adhesion is arrested, and the cells extravasate to their new environment and re-establish cell-cell adhesions (11). There are numerous various viewpoints regarding the mechanism underlying metastatic tumor cell arrest to distant organ sites $(12,13)$. Angiogenesis is necessary for cancer cell proliferation and metastasis (12); however, there remain a number of unknown mechanisms, including the precise mechanism underlying how cells alternate between exhibiting primary and metastatic properties.

Therefore, in the present study, gene expression profiles were used to further investigate the metastatic mechanism of OS. The GSE49003 dataset considered in the present study was previously analyzed by Diao et al (14) to identify differentially expressed genes (DEGs) between metastatic and non-metastatic patients with OS, and crucial microRNAs associated with OS metastasis, by merging data from different metastatic or non-metastatic OS cell lines. However, different types of metastatic OS may be regulated by different molecular mechanisms. In addition, transcription factors (TFs) may also serve a vital role in this pathomechanism. Therefore, the dataset was reanalyzed in the present study to emphasize the different mechanisms of different metastatic OS cell lines. 


\section{Materials and methods}

Gene expression profile data. The raw expression data (dataset GSE49003; http://www.ncbi.nlm.nih.gov/geo/query/acc. cgi?acc $=$ GSE49003), as provided by Endo-Munoz et al on July 18, 2013, were used in the present study. The microarray expression profile was obtained from two metastatic OS cell lines and two non-metastatic OS cell lines. The metastatic KHOS and KRIB cell lines and the non-metastatic HOS cell line were used with three-duplicated samples, and gene expression data from each of these cell lines were used. The platform of this dataset was GPL6847 Illumina HumanHT-12 V3.0 expression beadchip (Illumina Inc., San Diego, CA, USA).

Data preprocessing and DEG screening. The Limma package (15) in Bioconductor was used to add probe annotation files for each Illumina chip in order to preprocess the expression profile. Background correction, quantile normalization and probe summarization were performed to generate the gene expression data matrix.

DEGs between KHOS and HOS and between KRIB and HOS were determined using the Limma package. The differential expression of genes were analyzed by Student's t-test, and those with a false discovery rate adjusted P-value of $<0.01$ and Ifold changel $\geq 2$ were screened.

Functional enrichment analysis of DEGs. The Gene Ontology (GO) (16) project was established for gene classifications by molecular function, biological process (BP) and cellular component. DEGs of KHOS vs. HOS and KRIB vs. HOS were functionally enriched by GO. In addition, the Kyoto Encyclopedia of Genes and Genomes (KEGG) (17), which has a become a major database resource for understanding high-level functions of genes, was utilized. The default threshold of $\mathrm{P}<0.01$ was selected for the hypergeometric enrichment test.

Protein-protein interaction (PPI) network construction and subnetwork mining. The Search Tool for the Retrieval of Interacting Genes/Proteins is a biological database that provides known and predicted PPIs (13). The tool was applied in the present study to identify interacting protein pairs between DEGs with a PPI score of 0.9. Subsequently, Cytoscape software version 2.8.0 (18) was used to visualize the constructed PPI network. Subnetworks (modules) with a hypergeometric P-value $<0.05$ were identified by the ClusterONE plugin (19) from Cytoscape. Furthermore, the Database for Annotation, Visualization and Integrated Discovery $(20,21)$ was utilized to perform the KEGG pathway cluster analyses of DEGs in modules with $\mathrm{P}<0.05$.

Overlapping gene analysis. Overlapping DEGs that were upregulated in KHOS and KRIB cells compared with HOS cells were identified, and downregulated DEGs that were common in KHOS and KRIB cells were identified. Thereafter, KEGG signaling pathways of these two types of overlapping genes were enriched.

Furthermore, based on the regulatory association between TFs and target genes recorded in the University of California Santa Cruz (UCSC) (22) database, regulatory associations between TFs and their target DEGs were identified.

\section{Results}

DEGs of various groups. A total of 1,552 (711 downregulated and 841 upregulated) and 1,330 DEGs (570 downregulated and 760 upregulated) were obtained from the KHOS vs. HOS and KRIB vs. HOS comparisons, respectively.

Functional enrichment analyses of DEGs. Significant enriched terms of GO and KEGG pathway enrichment analyses in KHOS and KRIB groups are presented in Tables I and II, respectively. Upregulated genes of KHOS were associated with GO-BP terms of anatomical structure morphogenesis and cellular response to extracellular stimulus, and the downregulated genes were enriched in BP terms of multicellular organismal development and anatomical structure morphogenesis. Using KEGG, focal adhesion, axon guidance and peroxisome proliferator-activated receptor signaling pathways were significantly enriched by upregulated genes of KHOS, and pathways in cancer, tight junctions and cell adhesion molecules were enriched by downregulated DEGs in KHOS cells (Table I). GO and pathway enrichment analyses of KRIB were similar to those of KHOS, and the significantly enriched GO term of KRIB overexpressed genes was anatomical structure morphogenesis, and the significantly enriched pathway was focal adhesion (Table II).

PPI network and subnetwork. A total of 856 pairs of interacting proteins and 495 nodes were noted in the PPI network of KHOS (Fig. 1A). The top 6 nodes with a greater degree of connectivity compared with the others were jun proto-oncogene (JUN; degree $=33$ ), vascular endothelial growth factor A (VEGFA; degree=29), epidermal growth factor receptor (EGFR; degree=23), cyclin-dependent kinase inhibitor 1A (degree=19), interleukin 6 (IL-6; degree=19) and signal transducer and activator of transcription 3 (STAT3; degree $=17$ ). Furthermore, two modules with $\mathrm{P}<0.05$ were obtained (Fig. 1B and C). A total of 9 genes, including 7 collagen molecules and 2 integrin family members involved in module 1 were significantly enriched in terms of ECM-receptor interaction and focal adhesion.

There were 719 interacting pairs and 397 nodes of the PPI network of KRIB (Fig. 2). VEGFA (degree=29), Fyn oncogene related to Src, FGR, Yes (degree=25), JUN (degree=23), EGFR (degree $=22$ ) and IL-6 (degree=19), were the top 5 hub nodes. In addition, one module was screened (Fig. 2), and the significant pathways enriched by its genes were ECM-receptor interaction and focal adhesion.

$K E G G$ pathway enrichment and transcriptional regulatory network analysis of overlapping DEGs. A total of 421 upregulated and 595 downregulated overlapping genes were obtained. Pathway of focal adhesion, glutathione metabolism and endocytosis were enriched by their upregulated genes, whereas pathways in cancer, focal adhesion and cell adhesion molecules were enriched by downregulated genes. In addition, the mitogen-activated protein kinase (MAPK) and transforming growth factor $\beta$ (TGF- $\beta$ ) signaling pathways were enriched by overlapping genes with decreased expression levels (Table III).

Fig. 3 demonstrates the transactional regulatory network of overlapping DEGs. A total of 7 TFs, including activating 
Table I. Gene ontology and pathway enrichment analysis of genes in KHOS cells.

\begin{tabular}{|c|c|c|c|}
\hline Category identity & Name & Count & P-value \\
\hline \multicolumn{4}{|l|}{ Upregulated genes } \\
\hline GO:0009653 & Anatomical structure morphogenesis & 132 & $8.44 \times 10^{-7}$ \\
\hline GO:0031668 & Cellular response to extracellular stimulus & 20 & $1.75 \times 10^{-6}$ \\
\hline GO:0031669 & Cellular response to nutrient levels & 18 & $1.98 \times 10^{-6}$ \\
\hline GO:0071496 & Cellular response to external stimulus & 24 & $2.72 \times 10^{-6}$ \\
\hline GO:0072358 & Cardiovascular system development & 61 & $4.90 \times 10^{-6}$ \\
\hline Pathway: 4510 & Focal adhesion & 19 & $5.07 \times 10^{-4}$ \\
\hline Pathway:4360 & Axon guidance & 14 & $7.61 \times 10^{-4}$ \\
\hline Pathway:250 & Alanine, aspartate and glutamate metabolism & 6 & $1.66 \times 10^{-3}$ \\
\hline Pathway:3320 & PPAR signaling pathway & 9 & $2.08 \times 10^{-3}$ \\
\hline Pathway:4512 & ECM-receptor interaction & 10 & $2.35 \times 10^{-3}$ \\
\hline \multicolumn{4}{|c|}{ Downregulated genes } \\
\hline GO:0007275 & Multicellular organismal development & 310 & 0.00 \\
\hline GO:0009653 & Anatomical structure morphogenesis & 192 & 0.00 \\
\hline GO:0032502 & Developmental process & 338 & 0.00 \\
\hline GO:0044767 & Single-organism developmental process & 285 & 0.00 \\
\hline GO:0048468 & Cell development & 158 & 0.00 \\
\hline Pathway:5200 & Pathways in cancer & 35 & $2.61 \times 10^{-5}$ \\
\hline Pathway:5146 & Amoebiasis & 14 & $1.07 \times 10^{-3}$ \\
\hline Pathway:5144 & Malaria & 9 & $1.08 \times 10^{-3}$ \\
\hline Pathway: 4530 & Tight junction & 16 & $1.24 \times 10^{-3}$ \\
\hline Pathway:4514 & Cell adhesion molecules & 16 & $1.34 \times 10^{-3}$ \\
\hline
\end{tabular}

Count, number of genes enriched in the functional term; GO, Gene Ontology; PPAR, peroxisome proliferator activated receptor; ECM; extracellular matrix.

Table II. Gene ontology and pathway enrichment analysis of genes in KRIB cells.

\begin{tabular}{|c|c|c|c|}
\hline Category identity & Name & Count & P-value \\
\hline \multicolumn{4}{|l|}{ Upregulated genes } \\
\hline GO:0009653 & Anatomical structure morphogenesis & 106 & $3.88 \times 10^{-6}$ \\
\hline GO:0001944 & Vasculature development & 38 & $9.85 \times 10^{-6}$ \\
\hline GO:0010646 & Regulation of cell communication & 108 & $1.24 \times 10^{-5}$ \\
\hline GO:0071294 & Cellular response to zinc ion & 5 & $1.50 \times 10^{-5}$ \\
\hline GO:0023051 & Regulation of signaling & 107 & $1.88 \times 10^{-5}$ \\
\hline Pathway:4510 & Focal adhesion & 16 & $7.36 \times 10^{-4}$ \\
\hline Pathway:480 & Glutathione metabolism & 7 & $1.05 \times 10^{-3}$ \\
\hline Pathway:4360 & Axon guidance & 11 & $3.03 \times 10^{-3}$ \\
\hline Pathway:3320 & PPAR signaling pathway & 7 & $7.33 \times 10^{-3}$ \\
\hline Pathway:4520 & Adherens junction & 7 & $9.19 \times 10^{-3}$ \\
\hline \multicolumn{4}{|c|}{ Downregulated genes } \\
\hline GO:0000902 & Cell morphogenesis & 104 & 0.00 \\
\hline GO:0000904 & Cell morphogenesis involved in differentiation & 86 & 0.00 \\
\hline GO:0006928 & Cellular component movement & 132 & 0.00 \\
\hline GO:0007275 & Multicellular organismal development & 299 & 0.00 \\
\hline GO:0007399 & Nervous system development & 153 & 0.00 \\
\hline Pathway:4514 & Cell adhesion molecules & 19 & $1.14 \times 10^{-5}$ \\
\hline Pathway:5144 & Malaria & 11 & $1.74 \times 10^{-5}$ \\
\hline Pathway:5146 & Amoebiasis & 16 & $2.83 \times 10^{-5}$ \\
\hline Pathway:5200 & Pathways in cancer & 32 & $4.49 \times 10^{-5}$ \\
\hline Pathway:4512 & ECM-receptor interaction & 13 & $1.40 \times 10^{-4}$ \\
\hline
\end{tabular}

Count, number of genes enriched in the functional term; GO, Gene Ontology; PPAR, peroxisome proliferator activated receptor; ECM; extracellular matrix. 


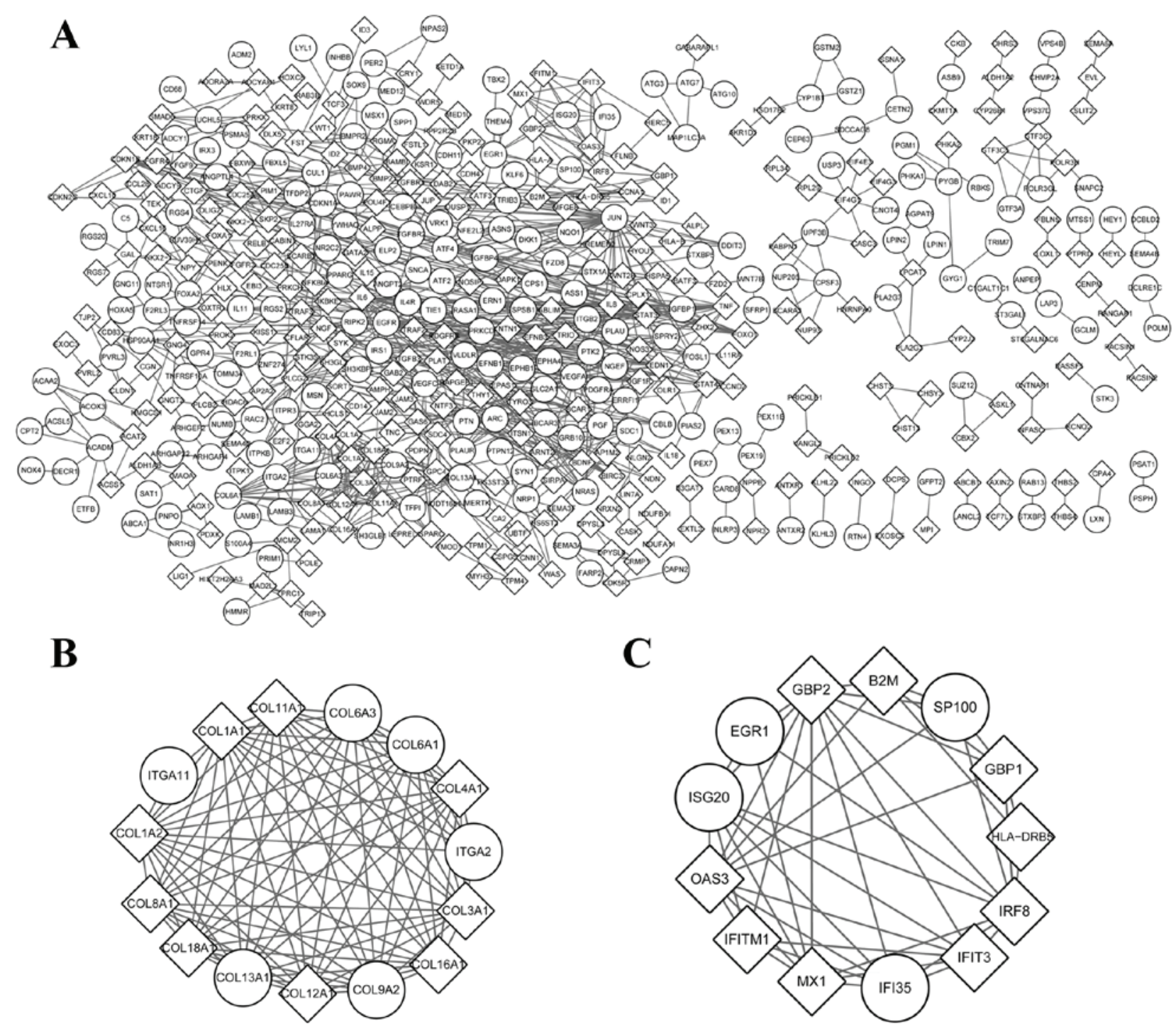

Figure 1. PPI network and subnetwork (module) of DEGs in KHOS cells. (A) PPI network. (B) Module 1. (C) Module 2. The diamond nodes denote the downregulated DEGs. The circular nodes represent the upregulated DEGs. Lines between nodes reveal the interactions between them. PPI, protein-protein interaction; DEGs, differentially expressed genes.

transcription factor 3, forkhead box (FOX)A1, STAT3, FOXA2, hes-related family bHLH transcription factor with YRPW motif 1 (HEY1), SUZ12 polycomb repressive complex 2 subunit and zinc finger ZZ-type containing 3 were identified. The first three of these were downregulated and the others were overexpressed.

\section{Discussion}

Due to the high risk for recurrence and the poor survival of metastatic OS patients (23), two different metastatic OS cell lines and one non-metastatic cell lines were used to investigate the mechanism underlying metastasis. The GO and pathway enrichment analysis results were similar of these two metastatic types of OS. Besides, a large number of overlapping genes, including $7 \mathrm{TFs}$, were obtained. These genes and biological functions may be important in OS tumor metastasis.

The most prominent node in the PPI networks of KHOS and KRIB was VEGFA, which is a growth factor that is activated in vasculogenesis, angiogenesis and endothelial cell growth (24). It is not only a commonly upregulated angiogenic factor during tumor growth, but also the most frequent lymphangiogenic factor contributing to the lymphatic metastasis (25). A previous study suggested that VEGFA promoted tumor growth and metastasis via the VEGF-VEGFR1 signaling pathway (26). In addition, EGFR, which is a member of the EGF family, was another common hub node with a high degree (27). It was revealed that EGFR participated in the promotion of cell division, migration and angiogenesis, and inhibited cell apoptosis (28). Furthermore, these two genes were enriched in the development and differentiation process, including anatomical structure morphogenesis and vasculature development. Yang et al (29) demonstrated that Twist, which is a master regulator of embryonic morphogenesis, contributed to metastasis by promoting an epithelial-mesenchymal transition (29). Therefore, tumor metastasis is the recapitulation of morphogenetic processes (30). Morphogenetic factors, including hepatocyte growth factor/scatter factor have 
Table III. Kyoto Encyclopedia of genes and genomes pathway of overlapping genes.

\begin{tabular}{|c|c|c|c|}
\hline Category identity & Name & Count & P-value \\
\hline \multicolumn{4}{|c|}{ Upregulated overlapping gene } \\
\hline 4510 & Focal adhesion & 13 & $2.96 \times 10^{-3}$ \\
\hline 480 & Glutathione metabolism & 5 & $3.11 \times 10^{-2}$ \\
\hline 4144 & Endocytosis & 10 & $3.18 \times 10^{-2}$ \\
\hline 4360 & Axon guidance & 8 & $3.46 \times 10^{-2}$ \\
\hline 4520 & Adherens junction & 6 & $3.67 \times 10^{-2}$ \\
\hline \multicolumn{4}{|c|}{ Downregulated overlapping gene } \\
\hline 5200 & Pathways in cancer & 27 & $2.52 \times 10^{-4}$ \\
\hline 4510 & Focal adhesion & 17 & $3.93 \times 10^{-3}$ \\
\hline 4514 & Cell adhesion molecules & 13 & $4.25 \times 10^{-3}$ \\
\hline 4512 & ECM-receptor interaction & 10 & $4.40 \times 10^{-3}$ \\
\hline 4010 & MAPK signaling pathway & 19 & $1.26 \times 10^{-2}$ \\
\hline 4350 & TGF- $\beta$ signaling pathway & 9 & $1.72 \times 10^{-2}$ \\
\hline
\end{tabular}

Count, number of genes enriched in the functional term; ECM, extracellular matrix; MAPK, mitogen-activated protein kinase; transforming growth factor- $\beta$.

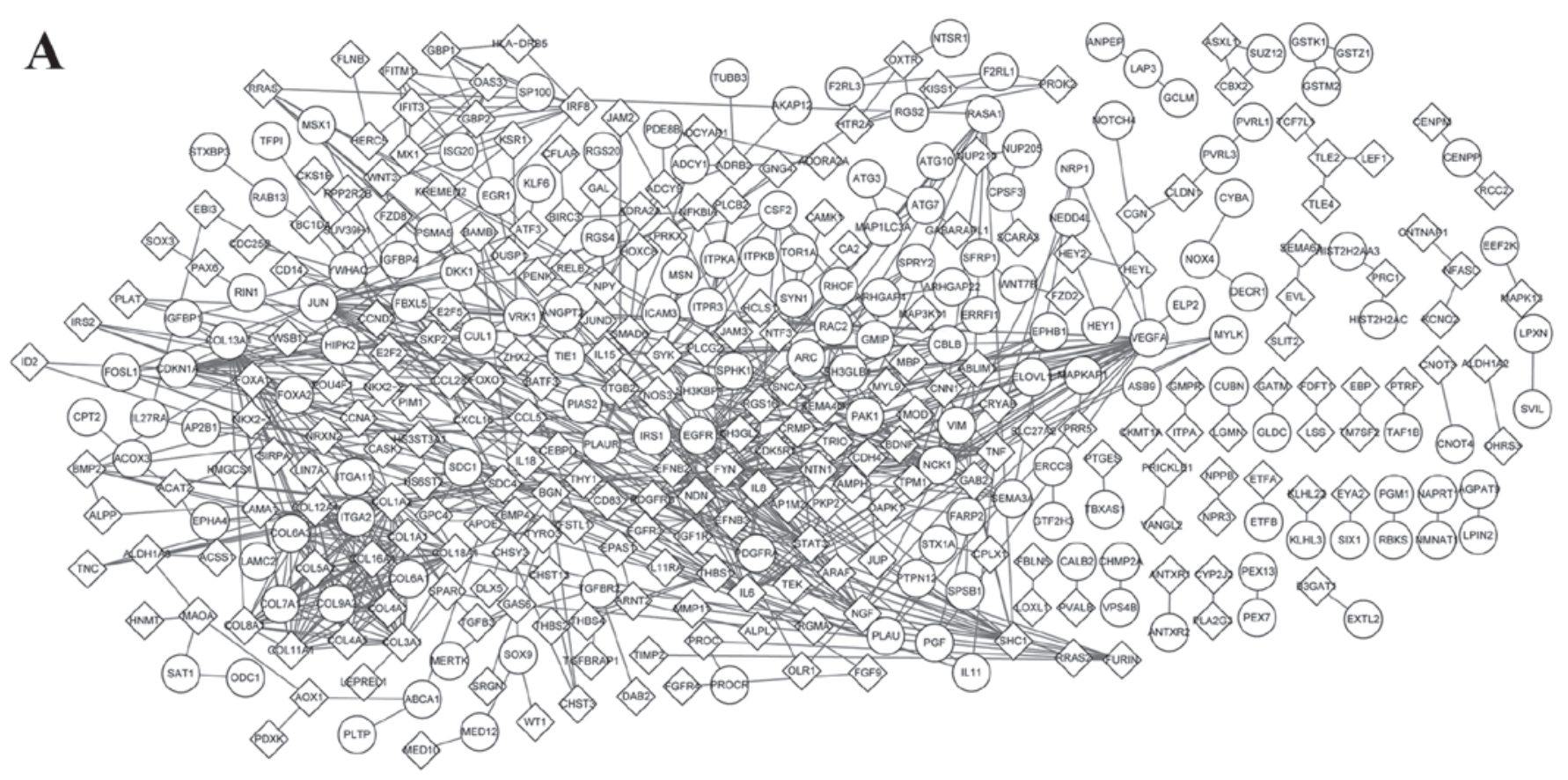

B

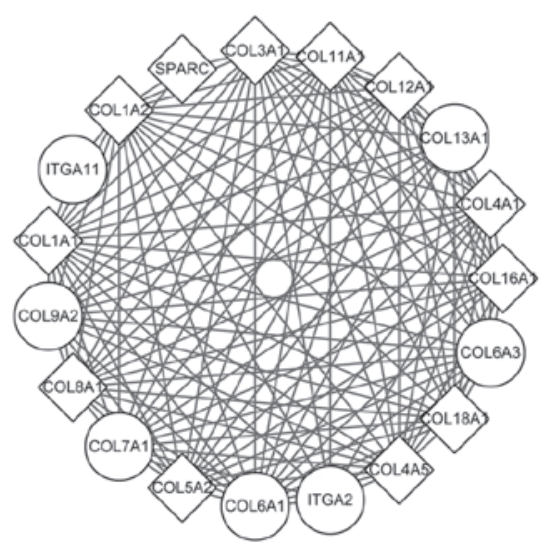

Figure 2. PPI network and subnetwork (module) of DEGs in KRIB cells. (A) PPI network. (B) Module one. The diamond nodes denote the downregulated DEGs. The circular nodes represent the upregulated DEGs. Lines between nodes reveal the interaction between them. PPI, protein-protein interaction; DEGs, differentially expressed genes. 


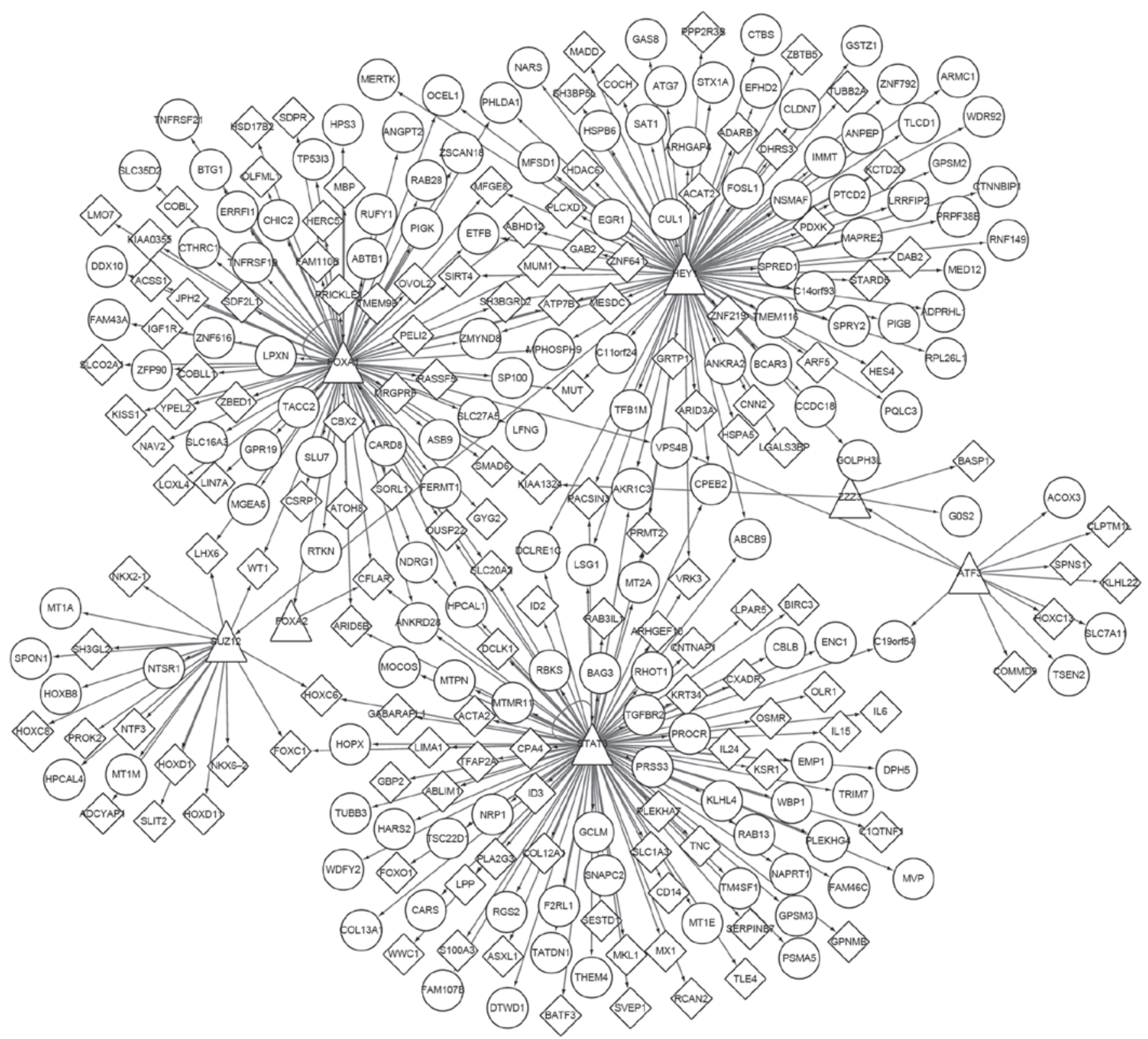

Figure 3. Transcription regulatory network of overlapping DEGs. The diamond nodes denote the downregulated DEGs. The circular nodes represent the upregulated DEGs. The triangular nodes reveal the transcription factors. DEGs, differentially expressed genes.

metastasis-promoting effects on breast carcinoma cells (31). Based on these studies, VEGFA and EGFR may be factors involved in the morphogenetic processes to promote tumor metastasis.

The KEGG pathway enrichment analysis results of module 1 of the KHOS group was similar to that of module 1 of the KRIB group. ECM-receptor interaction and focal adhesion were the observed pathways enriched by multiple collagen genes and integrin genes. These results were in accordance with previous studies $(32,33)$ and demonstrated the accuracy and practicability of them. Additionally, functional enrichment analysis of overlapping genes of these two group would be more direct to exhibit commonality of metastatic OS.

Focal adhesion was the significant pathway enriched by upregulated and downregulated overlapping genes. Furthermore, these downregulated genes remained enriched in signaling pathways that included the MAPK and TGF- $\beta$ signaling pathways. The former pathway serves an important role in the early metastasis of the tumor $(34,35)$, whereas the latter has positive and negative effects on carcinogenesis (36), and further investigation for is required to fully elucidate the molecular mechanism.

Finally, a total of 7 TFs, which regulated multiple genes, were screened. Among them, FOXA1, STAT3 and HEY1 were more important in the regulatory network. Candy et al (37) indicated that overexpressed HEY1 was a negative prognostic factor for colorectal cancer, and it is closely associated with vascular and perineural invasion and lymph node metastasis. Therefore, the upregulated HEY1 may be associated with OS metastasis. In addition, FOXA1 was revealed to be a participant in embryonic development, mediating tissue-specific gene expression in differentiated tissues (38). This gene encoded protein may be another important factor involved in the similar process of morphogenesis. 
In conclusion, recapitulation of morphogenetic processes, a disordered signaling system, and activated cell adhesion and migration were involved in the facilitated tumor metastasis. Furthermore, morphogenesis and vasculature development induced angiogenesis, and tumor growth and adhesion, or even the lymph node metastasis induced the metastatic OS. The present study further illustrated the OS tumorigenesis mechanism and displayed the crucial roles of genes such as VEGFA, EGFR and HEY1. The practical clinical significance has not yet been clearly described and requires further investigation.

\section{References}

1. Wu J, Hsieh MY, Wang Y, Roberts E, Vallejo D, Lee A and Ermel R: Antitumor properties of ouabain in lipid double emulsion in orthotopic canine osteosarcoma xenografted mouse model. Cancer Res 74: 5412, 2014.

2. Roos A, Yustein JT and Donehower L: Oncogenic role of Runx2 in the development of osteosarcoma. Cancer Res 73: 5036-5036, 2013.

3. Pompetti F, Rizzo P, Simon RM, Freidlin B, Mew DJ, Pass HI, Picci P, Levine AS and Carbone M: Oncogene alterations in primary, recurrent, and metastatic human bone tumors. J Cell Biochem 63: 37-50, 1996.

4. Oda Y, Naka T, Takeshita M, Iwamoto Y and Tsuneyoshi M: Comparison of histological changes and changes in $\mathrm{nm} 23$ and c-MET expression between primary and metastatic sites in osteosarcoma: A clinicopathologic and immunohistochemical study. Human Pathol 31: 709-716, 2000.

5. Liang S, Ren Z, Han X, Yang J, Shan L, Li L, Wang B, Zhang Q, $\mathrm{Mu} \mathrm{T}$, Chen K, et al: PLA2G16 expression in human osteosarcoma is associated with pulmonary metastasis and poor prognosis. PLoS One 10: e0127236, 2015.

6. Hughes DP: How the NOTCH pathway contributes to the ability of osteosarcoma cells to metastasize. In: Pediatric and Adolescent Osteosarcoma. Jaffe N, Bruland OS and Bielack S (eds). Vol 152. Springer, New York, NY, pp479-496, 2010.

7. Casar B, Rimann I, Kato H, Shattil SJ, Quigley JP and Deryugina EI: In vivo cleaved CDCP1 promotes early tumor dissemination via complexing with activated $\beta 1$ integrin and induction of FAK/PI3K/Akt motility signaling. Oncogene 33: 255-268, 2012.

8. Kim LC, Song L and Haura EB: Src kinases as therapeutic targets for cancer. Nat Rev Clin Oncol 6: 587-595, 2009.

9. Rubin EM, Guo Y, Tu K, Xie J, Zi X and Hoang BH: Wnt inhibitory factor 1 decreases tumorigenesis and metastasis in osteosarcoma. Mol Cancer Ther 9: 731-741, 2010.

10. Krishnan K, Khanna C and Helman LJ: The biology of metastases in pediatric sarcomas. Cancer J 11: 306-313, 2005.

11. Eccles SA and Welch DR: Metastasis: Recent discoveries and novel treatment strategies. Lancet 369: 1742-1757, 2007.

12. Fidler IJ: The pathogenesis of cancer metastasis: The 'seed and soil' hypothesis revisited. Nat Rev Cancer 3: 453-458, 2003.

13. Steeg PS: Tumor metastasis: Mechanistic insights and clinical challenges. Nat Med 12: 895-904, 2006.

14. Diao CY, Guo HB, Ouyang YR, Zhang HC, Liu LH, Bu J, Wang ZH and Xiao T: Screening for metastatic osteosarcoma biomarkers with a DNA microarray. Asian Pac J Cancer Prev 15 1817-1822, 2013.

15. Smyth GK: Limma: Linear models for microarray data. In: Bioinformatics and computational biology solutions using $\mathrm{R}$ and Bioconductor. Gentleman R, Carey VJ, Huber W, Irizarry RA and Dudoit S (eds). Springer, New York, pp397-pp420, 2005.

16. Ashburner M, Ball CA, Blake JA, Botstein D, Butler H, Cherry JM, Davis AP, Dolinski K, Dwight SS, Eppig JT, et al: Gene Ontology: Tool for the unification of biology. The Gene Ontology Consortium. Nat Genet 25: 25-29, 2000.

17. Kanehisa M and Goto S: KEGG: Kyoto encyclopedia of genes and genomes. Nucleic Acids Res 28: 27-30, 2000.
18. Shannon P, Markiel A, Ozier O, Baliga NS, Wang JT, Ramage D, Amin N, Schwikowski B and Ideker T: Cytoscape: A software environment for integrated models of biomolecular interaction networks. Genome Res 13: 2498-2504, 2003.

19. Nepusz T, Yu H and Paccanaro A: Detecting overlapping protein complexes in protein-protein interaction networks. Nat Methods 9: 471-472, 2012.

20. Huang da W, Sherman BT and Lempicki RA: Systematic and integrative analysis of large gene lists using DAVID bioinformatics resources. Nat Protoc 4: 44-57, 2008.

21. Huang da W, Sherman BT and Lempicki RA: Bioinformatics enrichment tools: Paths toward the comprehensive functional analysis of large gene lists. Nucleic Acids Res 37: 1-13, 2009.

22. Meyer LR, Zweig AS, Hinrichs AS, Karolchik D, Kuhn RM, Wong M, Sloan CA, Rosenbloom KR, Roe G, Rhead B, et al: The UCSC Genome Browser database: Extensions and updates 2013. Nucleic Acids Res 41 (Database issue): D64-D69, 2013.

23. PosthumaDeBoer J, Witlox MA, Kaspers GJ and Van Royen BJ: Molecular alterations as target for therapy in metastatic osteosarcoma: A review of literature. Clin Exp Metastasis 28: 493-503, 2011.

24. Liang D, Chang JR, Chin AJ, Smith A, Kelly C, Weinberg ES and Ge R: The role of vascular endothelial growth factor (VEGF) in vasculogenesis, angiogenesis, and hematopoiesis in zebrafish development. Mech Dev 108: 29-43, 2001.

25. Björndahl MA, Cao R, Burton JB, Brakenhielm E, Religa P, Galter D, Wu L and Cao Y: Vascular endothelial growth factor-a promotes peritumoral lymphangiogenesis and lymphatic metastasis. Cancer Res 65: 9261-9268, 2005.

26. Liu W, Xu J, Wang M, Wang Q, Bi Y and Han M: Tumor-derived vascular endothelial growth factor (VEGF)-A facilitates tumor metastasis through the VEGF-VEGFR1 signaling pathway. Int J Oncol 39: 1213-1220, 2011.

27. Zimmermann M, Zouhair A, Azria D and Ozsahin M: The epidermal growth factor receptor (EGFR) in head and neck cancer: Its role and treatment implications. Radiation Oncol 1: 11, 2006.

28. Italiano A, Saint-Paul MC, Caroli-Bosc FX, François E, Bourgeon A, Benchimol D, Gugenheim J and Michiels JF: Epidermal growth factor receptor (EGFR) status in primary colorectal tumors correlates with EGFR expression in related metastatic sites: Biological and clinical implications. Ann Oncol 16: 1503-1507, 2005.

29. Yang J, Mani SA, Donaher JL, Ramaswamy S, Itzykson RA, Come C, Savagner P, Gitelman I, Richardson A and Weinberg RA: Twist, a master regulator of morphogenesis, plays an essential role in tumor metastasis. Cell 117: 927-939, 2004.

30. Berx G, Raspé E, Christofori G, Thiery JP and Sleeman JP: Pre-EMTing metastasis? Recapitulation of morphogenetic processes in cancer. Clin Exp Metastasis 24: 587-597, 2007.

31. Meiners S, Brinkmann V, Naundorf $\mathrm{H}$ and Birchmeier W: Role of morphogenetic factors in metastasis of mammary carcinoma cells. Oncogene 16: 9-20, 1998.

32. Lu P, Weaver VM and Werb Z: The extracellular matrix: A dynamic niche in cancer progression. J Cell Biol 196: 395-406, 2012.

33. Felding-Habermann B: Integrin adhesion receptors in tumor metastasis. Clin Exp Metastasis 20: 203-213, 2003.

34. Khanna C, Wan X, Bose S, Cassaday R, Olomu O, Mendoza A, Yeung C, Gorlick R, Hewitt SM and Helman LJ: The membranecytoskeleton linker ezrin is necessary for osteosarcoma metastasis. Nat Med 10: 182-186, 2004.

35. Wan X, Mendoza A, Khanna C and Helman LJ: Rapamycin inhibits ezrin-mediated metastatic behavior in a murine model of osteosarcoma. Cancer Res 65: 2406-2411, 2005.

36. Derynck R, Akhurst RJ and Balmain A: TGF-beta signaling in tumor suppression and cancer progression. Nat Genet 29: 117-129, 2001.

37. Candy P, Phillips MR, Redfern AD, Colley SM, Davidson JA, Stuart LM, Wood BA, Zeps N and Leedman PJ: Notch-induced transcription factors are predictive of survival and 5-fluorouracil response in colorectal cancer patients. Br J Cancer 109: 1023-1030, 2013.

38. Bernardo GM and Keri RA: FOXA1: A transcription factor with parallel functions in development and cancer. Biosci Rep 32: 113-130, 2012. 